\title{
Cadena de valor de las empresas de producción y procesamiento de cárnicos de la ciudad de Cuenca
}

URL: http://revistas.uta.edu.ec/erevista/index.php/bcoyu/article/view/717 DOI: http://dx.doi.org/10.31164/bcoyu.22.2019.717

María González- Calle ${ }^{1}$; Juan Maldonado- Matute ${ }^{2}$; Katheryn Sinmaleza- Quezada ${ }^{3}$

Fecha de recepción: 26 de abril de 2019

Fecha de aceptación: 4 de julio de 2019

\section{Resumen}

La presente investigación tiene como objetivo analizar la cadena de valor de las empresas de producción y procesamiento de cárnicos en Cuenca. Se aplicó la metodología cualitativa presentada por Hochrein, Glock, Bogaschewsky y Heider (2015). Además de la aplicación de cuestionarios y entrevistas para el levantamiento de la cadena de valor. En conclusión, la cadena de valor presento debilidades y fortalezas en todos los eslabones, pero el que más impacto negativo tiene es el eslabón de recursos humanos, las empresas deben tomar acciones de mejora para convertir estas desventajas en oportunidad de mejorar.

Palabras clave: Cadena de valor, competitividad, sector cárnico

\section{Abstract}

The objective of this research is to analyze the value chain of meat production and processing companies in Cuenca. The qualitative methodology presented by Hochrein, Glock, Bogaschewsky and Heider (2015) was applied. In addition to the application of questionnaires and interviews for the lifting of the value chain. In conclusion, the value chain presented weaknesses and strengths in all the links, but the one that has the most negative impact is the human resources link, companies must take improvement actions to turn these disadvantages into an opportunity to improve.

Keywords: Value chain, competitive, meet production sector

\section{Introducción}

El propósito de la investigación es de dotar de información verídica al sector de elaboración y conservación de carne del Ecuador y de manera más puntual a la ciudad de Cuenca, para de esta manera apoyar la toma de decisiones empresariales y fortalecer las estrategias competitivas de las empresas del sector. Para entender la manera en que se desenvuelven las empresas de un sector se debe identificar las fuentes de ventajas competitivas, es por ello que se analiza de forma sistemática todas las actividades que una empresa desempeña, de esta manera se puede obtener una visión integral de ella e identificar sus fortalezas y debilidades. Resulta importante analizar la cadena de valor, debido a que, permite que las organizaciones conozcan su posición en relación a sus competidores, ver a la empresa dividas por eslabones permite identificar fuentes de valor que pueden convertirse en ventajas competitivas.

Porter (1985), en su obra Competitive Advantage - Creating and Sustaining Superior Performance, introdujo el concepto de cadena de valor como una herramienta que permite identificar las actividades relevantes de una empresa para luego clasificarlas de acuerdo con su importancia estratégica. Según el autor, la cadena de valor de una empresa forma parte de un sistema más grande denominado sistema de valor, un sistema en la que los proveedores, los canales de distribución y los clientes cuentan también con sus propias cadenas de valor.

Morillo \& Márquez (2013) realizan un análisis de la cadena de valor en el sector alimentos y bebidas donde se aprecia las principales estrategias que realizan las pequeñas y medianas industrias del sector; en este estudio se manifiesta las fuertes debilidades de las empresas del sector en cuanto a su poder de negociación con distribuidores y proveedores, e identifica estrategias como los esfuerzos que realizan para la prevención de errores, reorganización de actividades y cambios de materiales, estas se convierten en ventajas de diferenciación y reducción de costos que permite incrementar las ganancias y lograr un desempeño superior frente a sus competidores. Michael Jensen en su artículo "Value Maximization, Stakeholder Theory, and the Corporate Objective Function", propuso la teoría ilustrada de las partes interesadas (Enlightened Stakeholder Theory) en la que afirma que las empresas cuyas actividades únicamente persiguen el beneficio de sus socios, y no la maximización del valor de todas sus partes interesadas, tienen menor probabilidad de que sean exitosas. El autor de esta teoría afirma que los bajos salarios y las deficientes condiciones de trabajo llevan a las empresas a disminuir su productividad y su valor (Jensen, 2000).

\section{Marco teórico}

El fundamento de la cadena de valor es el lograr que las unidades de negocio desarrollen una ventaja competitiva Porter plantea estrategias genéricas basadas en costo, diferenciación o en ambas que permiten generar una posición competitiva ante sus rivales. Delimitar el termino ventaja competitiva resulta fundamental para que las empresas identifiquen aquellas fuentes de valor. Para ello coincidiendo con lo expuesto por Porter de que "La ventaja competitiva no puede ser comprendida viendo a una empresa como un todo" (Porter, 1991, pág. 3) es necesario indagar más allá de lo que la sociedad o cualquier grupo de interés pueden percibir a simple vista. Obtener una ventaja competitiva consiste en saber encajar las diferentes estrategias, es decir, viene a ser la forma en que los administradores estructuran la cadena de valor de la compañía mediante diferentes estrategias, de esta manera crean competencias distintivas que sirven para obtener un alto nivel de eficacia, eficiencia, innovación, calidad y capacidad de respuesta con el cliente obteniendo costos menores y diferenciándose de los rivales.

Ahora con el concepto de ventaja competitiva y al entender la importancia de desintegrar a la empresa en partes para identificar su ventaja es importante conocer la cadena de valor. Todos los productos y servicios se transforman a través de una línea de actividades relacionadas entre sí, por ello es importante para una organización conocer los diferentes procesos y actividades y coordinarlas. Pero esto no es suficiente para encontrar una ventaja estratégica, porque el punto faltante es el agregar valor a cada uno de los procesos y por esta razón se crea la cadena de valor. (Chase \& Jacobs, 2011). Afirmando lo expuesto por el autor, Francés considera que la cadena de valor permite saber en qué posición se encuentra la empresa con relación a sus competidores, además de ser una fuente para las futuras decisiones a tomar con el fin de crear una ventaja competitiva. (2006)

Distintos autores presentan diferentes perspectivas en cuanto al término cadena de valor a continuación en la tabla 1 se resumen las principales acotaciones.

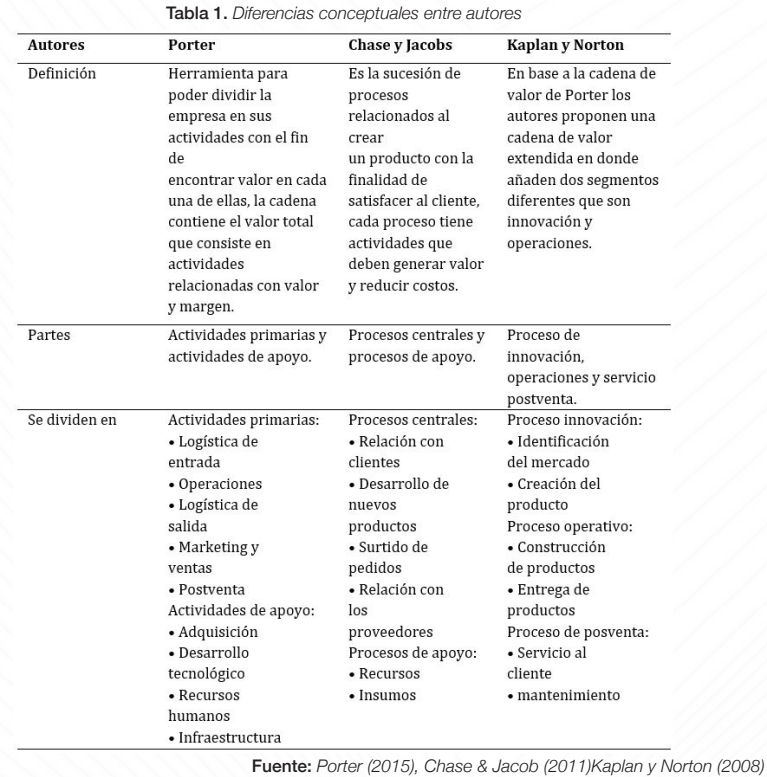

\section{Metodología}

La elección del sector objeto de este estudio fue definido mediante un análisis elaborado en varios ámbitos estratégicos como son: el nivel de ventas anuales, el número de trabajadores, la representatividad del sector, entre otros, todo esto enmarcado en la ciudad de Cuenca. La base en la que sustenta los ingresos, el número de empleados, y su información de contacto se consiguió a través del Servicio de Rentas Internas.

La metodología utilizada para revisión bibliográfica en la que sustenta la investigación se basa en la metodología cualitativa de revisión literaria sistémica presentada por Hochrein, Glock, Bogaschewsky y Heider (2015).El proceso de selección de la información de esta metodología contempla tres dimensiones explicadas en la figura 1. 


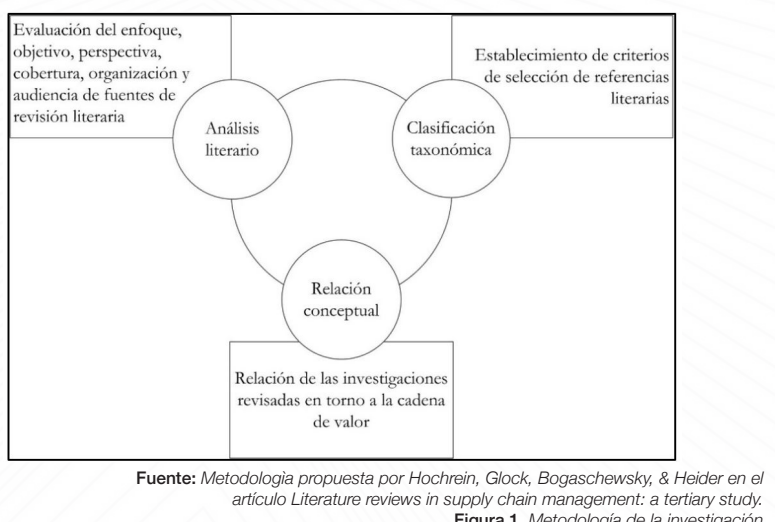

Figura 1. Metodología de la investigación

El análisis literario evalúa que la organización de los trabajos responda a la metodología conceptual, es decir, que las categorías guarden relación con el modelo de cadena de valor de Porter.

En una segunda etapa, con base en la información obtenida en la primera, se estructuró un cuestionario que sirvió para obtener información de las actividades de las organizaciones del sector y analizar los nueve eslabones de la cadena de valor de forma profunda. Para validar este instrumento como herramienta de análisis de la cadena de valor se practicó un pilotaje en empresas del sector del calzado de la ciudad de Cuenca. Es relevante indicar que la herramienta se considera adecuada para ser aplicada en cualquier sector productivo con pequeñas modificaciones de enfoque. El cuestionario consta de nueve secciones con un total de ciento dos preguntas, en su mayoría de carácter dicotómico, que abordan los diferentes aspectos de la cadena de valor, el cuestionario se aplicó mediante entrevistas y conversatorios con los representantes de las empresas o los gerentes de sus áreas estratégicas.

Con la información obtenida de las diferentes organizaciones colaboradoras, se identificó la situación actual de la cadena de valor de las industrias del sector de producción y procesamiento de cárnicos de la ciudad de Cuenca identificando fortalezas y debilidades del sector. Esta investigación no presenta resultados individuales de cada empresa y respeta acuerdos de confidencialidad y uso de información.

\section{Resultados}

La sección de resultados describe las actividades de soporte y las actividades primarias de cada uno de los grupos de estudio.

La estructura empresarial familiar es una de las fuerzas productivas y de organización más antiguas del mundo, en muchos casos las empresas evolucionan de un negocio pequeño a grandes compañías determinada por la administración de varias generaciones del grupo familiar, tal es el caso de Wal-Mart, Lego, Volkswagen entre otras, en Ecuador no es la excepción en este tema ya que entre un 90,5\% de empresas registradas en la Superintendencia de Compañías pertenecen a este tipo de estructura según un estudio realizado por la Universidad de Especialidades Espíritu Santo (UEES) y en el caso de las empresas obligadas a llevar contabilidad asociadas al sector de cárnicos en la ciudad de Cuenca los resultados afirman tal escenario, el 85,7\%, son empresas de tipo familiar.

En cuanto al nivel de ventas el $66,67 \%$ de las empresas tienen ventas anuales sobre el millón de dólares mientras que el 16,67\% venden entre \$ 500 mil y \$ 1 millón, y el porcentaje restante venden entre 100 mil y \$ 500 mil dólares.

Las empresas de cárnicos en la ciudad de Cuenca generan un gran número de plazas de empleo como se muestra en la figura 3 un total de 885 empleados en las 7 empresas encuestadas, distribuidos 464 en planta, 257 en administración y 164 en la fuerza de ventas y que realizan las actividades que se muestran en la figura 2.

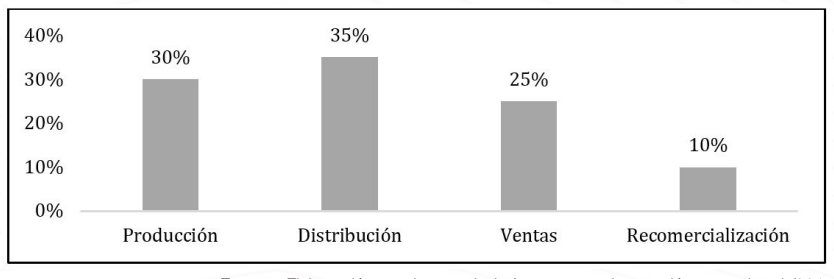

Fuente: Elaboración propia a partir de (encuestas, observación, entrevistas) (2017) Figura 2. Actividades que realizan las empresas obligadas a llevar contabilidad del sector cárnicos
Con respecto al eslabón infraestructura empresarial, se puede apreciar que e $71,43 \%$ de las empresas cuenta con un plan estratégico, organigrama definido, misión y visión y un plan operativo anual. El 85,7 \% cuenta con un sistema de gestión de calidad (SGC) y únicamente la mitad de éstas cuenta con una certificación, esto se debe a que existen empresas que, a pesar de no estar certificadas, manejan sus procesos y controles siguiendo los lineamientos de algún sistema de gestión, generalmente ISO 9001 o BPM. En el caso de sistemas de gestión ambiental y sistemas de salud y seguridad ocupacional, el $57,14 \%$ de las empresas, ha optado por seguir las recomendaciones que plantea BPM, mas no poseen certificaciones específicas en estas áreas ver figura 3.

Los organismos de control respecto a los productos alimenticios en la ciudad de Cuenca están enfocados en atacar a las enfermedades trasmitidas por los alimentos ya que estos representan un problema a nivel mundial, la OMS registra 2000 millones de casos de enfermedades trasmitidas por alimentos. La carne de res es una de las principales vías de trasmisión de enfermedades para los seres humanos debido a su composición orgánica que permite la proliferación de bacterias.

Las empresas de cárnicos han optado por seguir las normas que han sido desarrolladas con el objetivo de asegurar que los alimentos mantengan su inocuidad en toda su cadena de valor como, por ejemplo: Buenas Prácticas de Manufactura, el sistema de Análisis de Peligrosos y Puntos Críticos de Control.

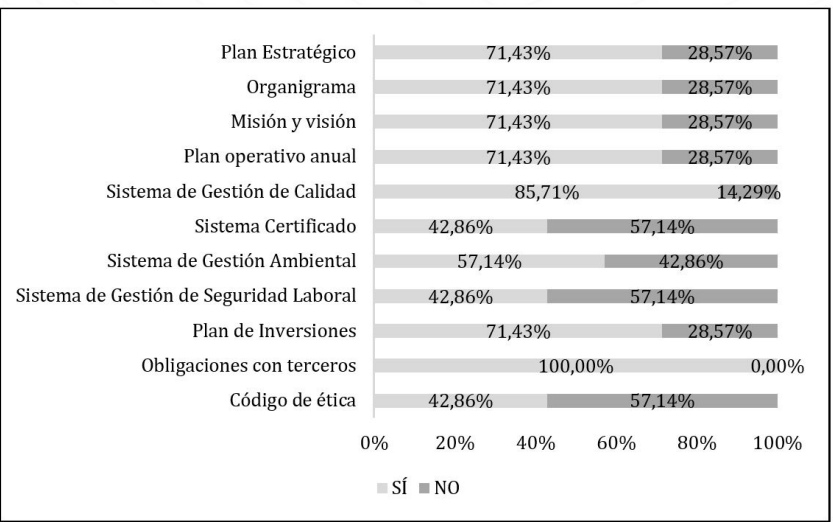

Fuente: Elaboración propia a partir de (encuestas, observación, entrevistas) (2017) Figura 3. Eslabón de infraestructura de las empresas obligadas a llevar contabilidad

La tecnología en la actualidad es un recurso fundamental para todo tipo de empresas ya sea por su tamaño o sector, la tecnología elimina barreras internas en las organizaciones mediante los sistemas de comunicación y datos en tiempo real. Las empresas que se encuentran en crecimiento deben sobrellevar las barreras que se les presenta al momento de adquirir dicho recurso para adaptarse a los cambios económicos, procesos y sobre todo mantener la competencia.

Todas las empresas definen objetivos anuales, sin embargo, en el 42,9 \% de los casos estos no se conectan con la misión y visión; a pesar de esto, el 71,4% dispone de mecanismos para evaluar su cumplimiento. En el último año todas las empresas han invertido en maquinaria, no obstante, no todas lo han hecho en tecnología o infraestructura. Todas las organizaciones manejan políticas de responsabilidad social empresarial en especial en el ámbito de cooperación y ayuda social.

En lo respecto al eslabón de recursos humanos, solamente el 28,57 \% de las empresas ha creado políticas de $\mathrm{RRHH}$, el $85,71 \%$ ha definido procesos de selección y, políticas de seguridad y salud ocupacional, el 71,43\% ha elaborado planes de capacitación, planes de evaluación de desempeño y políticas para incluir a los colaboradores en los procesos de toma de decisiones. En lo que respecta clima laboral, el 57,14\% de las empresas evalúa a sus empleados sobre este aspecto y también el $57,14 \%$ ha diseñado un plan de incentivos para sus colaboradores. Adicional a esto, la totalidad de las empresas dispone de planes de inducción y perfiles de cargos definidos, aunque solo el 42,86\% cuenta con métodos para evaluar los perfiles ver figura 4. 


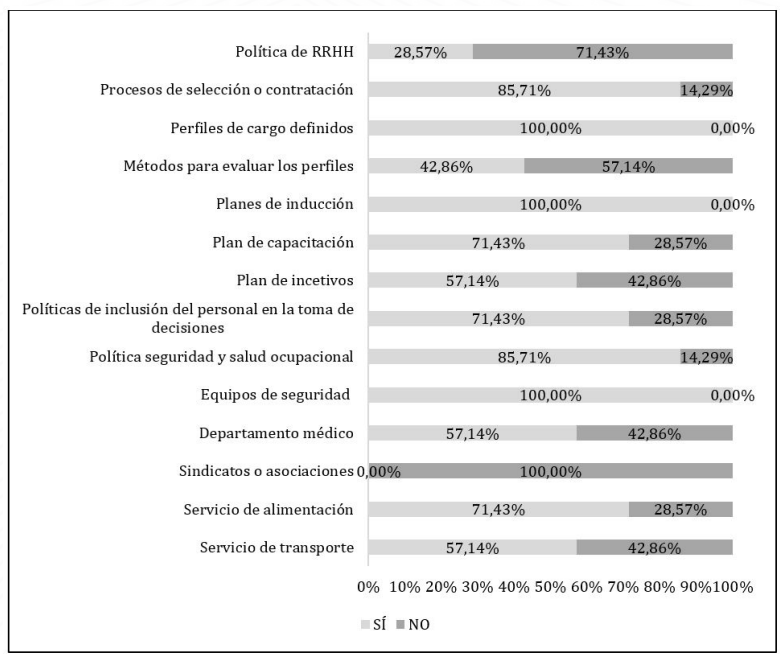

Fuente: Elaboración propia a partir de (encuestas, observación, entrevistas) (2017) Figura 4. Recursos humanos de las empresas obligadas a llevar contabilidad

En el ámbito tecnológico, todas las empresas emplean las tecnologías de la información (TIC) tanto en los procesos de comunicación como en los procesos de marketing y ventas: el $100 \%$ posee servicios de internet y utiliza el correo electrónico para comunicación interna con sus clientes y proveedores; el 42,86 \% posee página web. La página web presenta información referente a la empresa y se brinda la opción de servicio al cliente, adiciona información de sus productos e información nutricional y presentaciones, también brindan la opción de consulta de facturas electrónicas, solamente una entrega la información nutricional de sus productos y ofrece el servicio de chat en línea, ninguna organización ha incorporado la opción de compras online ver figura 5.

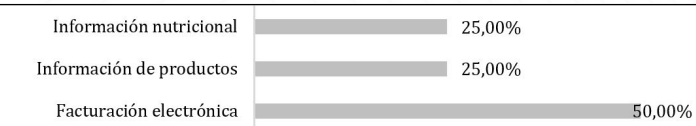

$$
0 \% \quad 10 \% \quad 20 \% \quad 30 \% \quad 40 \% \quad 50 \%
$$

Fuente: Elaboración propia a partir de (encuestas, observación, entrevistas) (2017) Fuente: Elaboración propia a partir de (encuestas, observación, entrevistas) (2017)
Figura 5. Funcionalidad de la página web de las empresas obligadas a llevar contabilidad del sector de cárnicos Referente a la gestión de compras como muestra la Figura 6 el 57,14\% de las empresas cuenta con procesos de compras definidos y mecanismos para seleccionar a sus proveedores. El 85,71\% realiza controles de calidad al momento de recibir sus pedidos y además exigen a sus proveedores el cumplimiento de normas o estándares establecidos por la empresa.

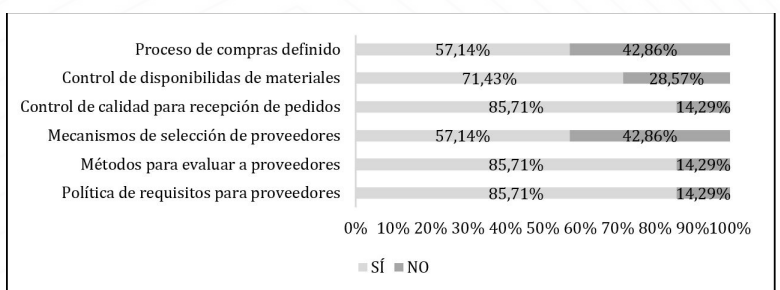

Fuente: Elaboración propia a partir de (encuestas, observación, entrevistas) (2017)

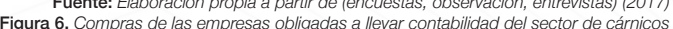

Uno de los elementos fundamentales para la cadena de valor de una empresa es la logística, este elemento cambia de manera drástica las operaciones que maneja cualquier tipo de organización, en el sector cárnicos el manejar de manera correcta representa no solo una fortaleza en la cadena antes mencionada, sino que abarca sustancialmente un rendimiento eficaz en la cadena de suministros, es decir que los productos lleguen al lugar correcto en el tiempo correcto.

Cuatro empresas indicaron que existe dificultad para conseguir ciertas materias primas e insumos, esto debido principalmente a que el mercado nacional no abastece las necesidades del sector, no existen ciertos insumos o la calidad existente no es la requerida, también la estacionalidad de ciertas materias primas juega un papel importante en este aspecto.

En lo relacionado a las actividades primarias, se iniciará describiendo la situación de la logística interna. El $85,71 \%$ de las organizaciones cuentan con plan de logística y métodos de control de entrada de la materia prima. Todas las organizaciones cuentan con áreas adecuadas para el almacenamiento de insumos, materias primas y productos terminados, también el 100\% de las empresas tienen conocimiento de su inventario real y teórico. y las variaciones al contrastar los dos tienen un error menor al 3 \% en la mayoría de los casos. Además, vemos que solo el $85,71 \%$ conoce los costos asociados al almacenamiento de inventario ver figura 7

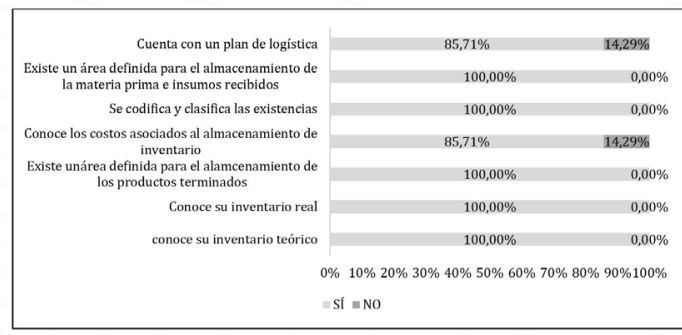

Fuente: Elaboración propia a partir de (encuestas, observación, entrevistas) (2017) Figura 7. Logística interna de las empresas obligadas a llevar contabilidad del sector de cárnicos Como muestra la Figura 8 para la gestión de la logística externa, 6 de las empresas disponen de medios propios para la distribución de sus productos y conocen los costos asociados a esta actividad, y dos cuentan con seguros para sus bodegas y productos terminados. El $57,14 \%$ de las industrias conoce los costos asociados con el mantenimiento y preservación de su producto terminado. Todas las empresas llevan el registro de productos entregados y el $85,71 \%$ lleva un registro y control de los productos no disponibles.

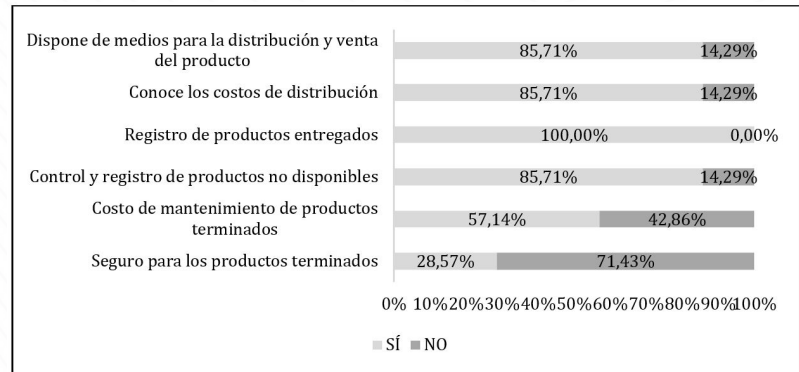

Fuente: Elaboración propia a partir de (encuestas, observación, entrevistas) (2017) Figura 8. Logística externa de las empresas obligadas a llevar contabilidad del sector de cárnico En relación a la forma en que llevan a cabo sus operaciones, todas las empresas poseen especificaciones técnicas de productos, realizan controles de calidad y llevan la trazabilidad de sus procesos productivos. El $85,7 \%$ de las organizaciones poseen técnicas para la planificación y programación de la producción, además tienen sus procesos estandarizados y documentados. Cinco de las organizaciones realizan medición del desempeño de sus procesos ver figura 9

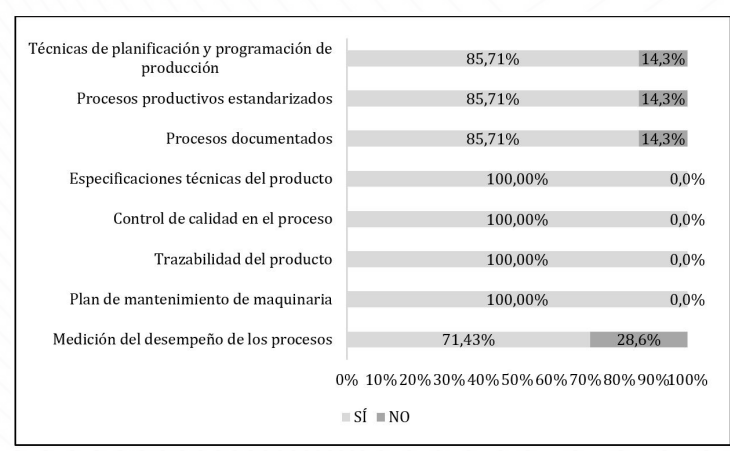

Fuente: Elaboración propia a partir de (encuestas, observación, entrevistas) (2017) Figura 9. Operaciones de las empresas obligadas a llevar contabilidad del sector de cárnico

Como se muestra en la Figura 10, todas las empresas tienen identificados sus costos de producción, en lo que se refiere a la capacidad, todas conocen su capacidad utilizada; sin embargo, dos empresas desconocen su capacidad real. En términos de desperdicios, el $85,71 \%$ conocen sus desperdicios y el $71,43 \%$ tienen mecanismos para disminuirlos. En todos los casos tienen mecanismos para disminuir el impacto ambiental generado por su actividad y dan tratamiento al producto caducado.

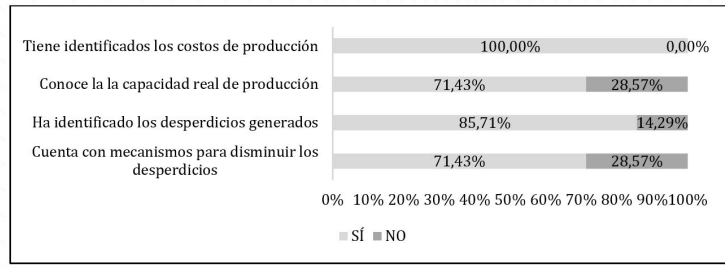

Fuente: Elaboración propia a partir de (encuestas, observación, entrevistas) (2017) Figura 10. Operaciones de empresas obligadas a llevar contabilidad del sector de cárnicos 
Para la gestión de marketing y ventas $85,7 \%$ de las empresas realizan investigación de mercados, tienen identificado claramente su segmento y consideran estrategias para su diversificación. La totalidad de las empresas cuentan con una imagen corporativa definida, mantiene un registro de sus clientes, poseen canales de comunicación permanente con éstos y tienen apertura para que la ciudadanía en general pueda conocer sus instalaciones y procesos productivos. Seis de las empresas tiene un registro de propiedad de marca, posee negocios complementarios y hace uso de redes sociales para sus procesos de comunicación y promoción. Los negocios complementarios principales son puntos de venta, delicatessen y cafetería; sobre el uso de redes sociales Facebook es utilizada por las seis empresas, Instagram por tres y Twitter por una. El 100\% de las empresas realiza publicidad de su marca y productos siendo los principales medios utilizados la radio y las redes sociales, dos de estas empresas han usado en algún momento publicidad por televisión, en menor medida se hace uso de medios impresos como periódicos, revistas o volantes. Todas las empresas cumplen con los requisitos legales de etiquetado proporcionando, información nutricional, de ingredientes, de uso de transgénicos y de características especiales de sus productos.

En lo que respecta al servicio post venta como se en la figura 11 el $71,4 \%$ de las empresas brindan servicio post venta a sus clientes, el 100\% ofrece garantía de sus productos, realiza un seguimiento de los reclamos que recibe y genera acciones de mejora ante estas situaciones, sin embargo, únicamente el $57,1 \%$ de las empresas guarda registros de los reclamos y mide la satisfacción de sus clientes. Únicamente 4 empresas cuantifican los costos asociados con los reclamos.

Potenciar la relación entre el consumidor y la marca es uno de los principales objetivos que el marketing maneja actualmente para las empresas, la idea de que el consumidor desee ser parte de todo el proceso que opera una compañía, obliga a estas a manejar diferentes planes y estrategias para controlar su mercadotecnia, los resultados levantados en el presente artículo indica que las empresas del sector en estudio un $85 \%$ conocen e implementan en alguna manera las técnicas y estrategias en relación a la materia de marketing.

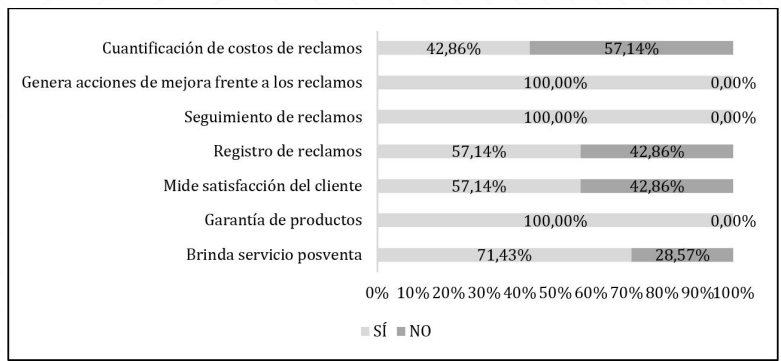

Fuente: Elaboración propia a partir de (encuestas, observación, entrevistas) (2017) Figura 11. Servicio de las empresas obligadas a llevar contabilidad del sector de cárnicos

Después del análisis de los nueve eslabones de la cadena de valor se puede resumir que el sector de producción y procesamiento de carne en la ciudad de Cuenca presenta las siguientes fortalezas y debilidades.

\section{Fortalezas:}

- Se manejan sistemas de gestión y planificación de objetivos.

- Existen una estructura organizacional bien definida.

- Llevan a cabo procesos de selección y contratación de personal.

- Poseen perfiles de cargo definidos.

- Proveen a sus colaboradores de equipos de seguridad según lo estipula la ley.

- Mantienen métodos y políticas para la evaluación y contratación de proveedores.

- Realizan de forma regular el control de calidad de su productos y procesos.

- Llevan la trazabilidad de su proceso productivo.

- En su mayoría poseen medios propios para la distribución y venta de sus productos.

- Cuentan con registro de propiedad de marca.

- Se realizan estudios de mercado y se conoce el segmento al cual se enfocan.

- Se utiliza la publicidad como un medio permanente para dar a conocer el negocio y sus productos.

- Todas las empresas ofrecen garantía en sus productos.

- Poseen una estructura tecnológica adecuada para sus actividades.

\section{Debilidades:}

- No se encuentran desarrolladas totalmente políticas para la gestión de seguridad laboral y conservación medio ambiental.

- No todas las empresas que poseen métodos para evaluar perfiles de cargos.

- Son pocas las empresas que han definido una política de recursos humanos.

- Casi no se consideran políticas de despido y fijación de sueldos.

- No se desarrollan planes de carrera de forma habitual.

- Ninguna empresa considera políticas de jubilación.

- Aún son escasas las políticas anti discriminación.

- No todas las empresas cuentan con seguro para sus bodegas y productos terminados.
- La medición de la satisfacción al cliente y el registro de reclamos se lleva a cabo en apenas un poco más de la mitad de las empresas.

\section{Conclusiones}

Una empresa o unidad de negocio que se prepara para enfrentar los retos futuros debe tener una ventaja competitiva sostenible en el tiempo para así contar con más posibilidades de lograr un mejor rendimiento en el sector donde opera. La investigación permitió identificar aquellas fortalezas y debilidades que se encuentran presentes en las empresas del sector de procesamiento y elaboración de cárnicos de la ciudad de Cuenca. Entre las principales estrategias se evidencia los constantes esfuerzos por mantener campañas de publicidad y crear una imagen corporativa, otra estrategia son las rigurosas especificaciones que piden a sus proveedores para que la materia prima cumpla con los estándares establecidos y así se logre un producto de calidad, también se evidencia una integración vertical de la cadena de suministros al haber empresas que se convierten en sus propios proveedores y gestionan sus propios criaderos. Estas estrategias se pueden convertir en ventajas competitivas de diferenciación de marca y de reducción de costos. Para futuros estudios se debería investigar la cadena de valor y su relación con la responsabilidad social empresarial como estrategia para la creación de ventajas competitivas.

Las personas obligadas a llevar contabilidad que forman parte del sector de producción y procesamiento de cárnicos de la ciudad de Cuenca presentan un gran potencial, según la Superintendencia de valores y seguros genera 1037 plazas de empleo, es por ello que dotar de información como la presente a los distintos agentes interesados es fundamental para apoyar a la toma de decisiones y al crecimiento del sector. puesto que podrán realizar un análisis de cómo se encuentra su empresa frente al sector y con esto mejorar su nivel de competitividad.

Desde el 2012 el sector cárnico en Ecuador empezó a sufrir impactos negativos ocasionados por las imperfecciones en la cadena de valor, la dificultad de acceder a una producción de economías de escala, y los precios poco flexibles y desfavorables, lo que obligó a los productores ganaderos a migrar a otras actividades que ofrezcan una mayor retribución. (Revista líderes, 2015). En concordancia con lo expuesto se identificó en el estudio que el eslabón más débil y el cual requiere una mejor gestión es el de recursos humanos, a partir de la información obtenida por la herramienta se evidencia que las empresas sí realizan prácticas de gestión enfocadas al proceso de inducción del personal o para cumplir con los ámbitos legales y no para mantener motivado al personal. Solo el $28,57 \%$ de las empresas cuentan con políticas de recursos humanos y el $42,60 \%$ realiza evaluaciones de desempeño. Existen herramientas que apoyan la gestión del departamento de recursos humanos, como un manual de funciones, perfiles de cargo, evaluaciones de desempeño, entre otras, y que las organizaciones pueden poner en práctica para mejorar el eslabón. Las sociedades del sector textil deben invertir en el proceso de gestión del talento humano afianzando el criterio expuesto por Martha Allen de que el capital humano motivado es estratégico para la generación de valor en las organizaciones. Las sociedades del sector de elaboración y procesamiento de carne deben tomar las debidas acciones de mejora y convertir estas desventajas en una oportunidad de mejorar.

\section{Referencias}

Chase, R., \& Robert, J. (2011). Administración de Operaciones Producción y Cadena de Suministros. México: McGRAW-HILL.

Francés, A. (2006). Estrategia y planes para la empresa: con el cuadro de mando integral.

Jensen, M. (2000). Value maximization and the corporate objective function Breaking the Code of Change, 12(2), 37-57. https://doi.org/10.2307/3857812 Hochrein, S., Glock, C., Bogaschewsky, R., \& Heider, M. (2015). Literature reviews in supply chain management: a tertiary study. Management Review Quarterly, 239-280.

Kaplan, R., \& Norton, D. (2008). Execution Premium. España: Ediciones Deusto.

Porter, M. (1991). Ventaja Competitiva, Creación y Sostenimiento de un Desempeño Superior. Buenos Aires, Argentina: Rei Argentina S.A.

Porter, M. (1985). Competitive Advantage - Creating and Sustaining Superior Performance. New York: The Free Press.

Porter, M. (2015). Ventaja Competitiva: creación y sostenimiento de un desempeño superior. Mexico: Patria.

Revista líderes. (2015). En ocho provincias se concentra el mayor consumo de cárnicos. Obtenido de https://www.revistalideres.ec/lideres/consumo-cárnicos-ecuador.html 